\section{Análise da qualidade da informação sobre causa básica de óbitos neonatais registrados no Sistema de Informações sobre Mortalidade: um estudo para Maceió, Alagoas, Brasil, 2001-2002}

\author{
Quality of information analysis on basic causes \\ of neonatal deaths recorded in the Mortality \\ Information System: a study in Maceió, \\ Alagoas State, Brazil, 2001-2002
}

Linda Délia C. O. Pedrosa 1,2

Silvia W. Sarinho 3

Manoelina R. Ordonha 1

\footnotetext{
1 Hospital Universitário Professor Alberto Antunes, Universidade Federal de Alagoas, Maceió, Brasil. 2 Escola de Ciências Médicas, Fundação Universitária de Ciências da Saúde de Alagoas Governador Lamenha Filho, Maceió, Brasil.

3 Centro de Ciências da Saúde, Universidade Federal de Pernambuco, Recife, Brasil.

Correspondência L. D. C. O. Pedrosa Hospital Universitário Professor Alberto Antunes, Universidade Federal de Alagoas.

Condomínio Aldebaran Beta, Quadra C, Lote 2, Maceió, AL 57080-900, Brasil.

lindapedrosa@uol.com.br
}

\begin{abstract}
Analysis of the quality of information on basic causes of neonatal deaths in Brazil is crucially important, since it allows one to estimate how many deaths are avoidable and provide support for policies to decrease neonatal mortality. The current study aimed to evaluate the reliability and validity of the Mortality Information System (MIS) for discriminating between basic causes of neonatal deaths and defining percentages of reducible causes. The basic causes of early neonatal deaths in hospitals in Maceió, Alagoas State, were analyzed, and the causes recorded in medical records were compared to the MIS data in order to measure reliability and validity. The modified SEADE Foundation and Wigglesworth classifications were compared to analyze the capacity for reduction of neonatal mortality. Maternal causes predominated in the medical records, as compared to respiratory disorders on the death certificates and in the MIS. The percentage of avoidable deaths may be much higher than observed from the MIS, due to imprecision in completing death certificates. Based on the MIS, the greatest problems are in early diagnosis and treatment of neonatal causes. However, the results show that the most pressing problems relate to failures in prenatal care and lack of control of diseases.
\end{abstract}

Neonatal Mortality; Underlying Cause of Death; Death Certificates; Mortality Registries
Introdução

Os óbitos infantis no Brasil têm se concentrado no período neonatal, onde causas de mais difícil redução necessitam, além de ações com tecnologia simples, de ações de maior elaboração operacional, em ambiente hospitalar complexo 1. O óbito neonatal é considerado um evento sanitário de alerta, por sofrer extrema influência da qualidade dos serviços oferecidos à população e sua análise é importante instrumento, desvendando limitações na atenção dedicada à gestante e ao neonato 2 .

A avaliação das informações sobre mortalidade deve considerar as circunstâncias clínicas que a propiciaram, além do preenchimento da declaração de óbito (DO) e codificação da causa básica 3 . O atual modelo de condições e causas da morte resultou de várias tentativas mundiais de definição da causa de óbito a ser tabulada nas estatísticas de mortalidade, denominada "causa básica" 4 . Esta origina uma sucessão de afecções conseqüenciais, a última chamada causa terminal ou direta. Também podem ser incluídas entre as condições e causas da morte as doenças que contribuíram para o óbito ${ }^{5}$.

A análise dos óbitos pode enfocar aspectos tais como o número de eventos, sexo, idade, local de ocorrência e a causa do óbito, que é considerada seu mais relevante aspecto 4 . A investigação das causas de óbitos pode fornecer informações sobre o acesso, adequação, qualidade dos servi- 
ços e, potencialmente, estimar sua efetividade 2 . Os óbitos também podem ser analisados sob a ótica de seu potencial de redução ou evitabilidade 6 , para a qual foram propostas diversas sistematizações de causas, dentre as quais a da Fundação Sistema de Análise dos Dados de São Paulo (Fundação SEADE) 6 e a classificação de Wigglesworth modificadas 7,8.

A classificação da Fundação SEADE modificada utiliza as causas constantes na décima revisão da Classificação Estatística Internacional de Doenças e Problemas Relacionados à Saúde (CID-10) 9 agrupadas de acordo com seu potencial de redução, apontando áreas onde a atenção pode ter falhado 10 . Wigglesworth 7 propôs um sistema de avaliação de óbitos perinatais modificado por Keeling et al. ${ }^{8}$ que, utilizando informações clínicas disponíveis nos prontuários e, prescindindo de necropsia, sinaliza áreas alvos de prevenção ${ }^{11,12}$.

A realização desse estudo se justifica por ser Maceió, capital do Estado de Alagoas, situado no Nordeste brasileiro, o Estado detentor dos mais elevados índices de mortalidade infantil do país, com taxa de mortalidade infantil de 55,57/ mil nascidos vivos em 2001 e de 52,64/mil nascidos vivos em 2002 13. Ao verificar a magnitude dos óbitos passíveis de serem evitados e a qualidade dessas informações, estudos semelhantes podem ser utilizados como subsídios para formulação de políticas públicas para redução dos óbitos infantis, em especial no período neonatal.

Objetivou-se avaliar a adequação das informações disponíveis no Sistema de Informações sobre Mortalidade (SIM), definindo sua confiabilidade e validade, através da análise da concordância da causa básica entre registros eletrônicos do SIM e DO preenchidas pelos médicos, tendo como padrão-referência causa básica definida pela análise dos prontuários; descrever e comparar o percentual de óbitos passíveis de redução, aplicando as classificações da Fundação SEADE modificada 6 e de Wigglesworth 7 modificada por Keeling et al. 8 .

\section{Material e método}

O desenho do estudo foi descritivo de validação. A população do estudo foi inicialmente definida em 453 óbitos neonatais precoces hospitalares, filhos de mães residentes em Maceió e informados pelo SIM como ocorridos entre 1o de janeiro de 2001 e 31 de dezembro de 2002. Dos 401 registros eletrônicos do SIM, obtidos após a limpeza do banco de dados, em 337 óbitos foram localizados os prontuários da mãe e neonato, proceden- do-se a comparação das fontes de informações. Esta sub-amostra foi utilizada na comparação das causas para análise da qualidade do SIM (concordância e sensibilidade). Os óbitos com peso ignorado ou inferior a $500 \mathrm{~g}$ foram excluídos da análise.

O cálculo do tamanho amostral 14 foi baseado na sensibilidade da DO em detectar causas neonatais de óbito de $83,3 \% 15$, admitindo-se erro $\alpha$ de $5 \%$ e poder de $80 \%$, resultou em amostra mínima de 334 óbitos, tendo-se optado por estudar todos os óbitos ocorridos.

Procedeu-se à validação dos endereços das mães pelo Código de Endereçamento Postal (CEP), seguindo protocolo de Guimarães et al. 16, e análise das DO fetais, para detectar erros de classificação, resultando na inclusão de um óbito neonatal encontrado entre os fetais. Também foi encontrada uma DO que não chegara à Secretaria Municipal de Saúde de Maceió, estando as três vias anexadas ao prontuário, no arquivo hospitalar, não constando este óbito dos eventos ocorridos em Alagoas naquele período. Foram excluídos óbitos fetais erroneamente classificados como: não fetais (12), óbitos domiciliares (1), óbito em adulto (1), os óbitos neonatais tardios (7) DO duplicadas (2) e filhos de mães residentes em outros municípios (27), erroneamente incluídos como óbitos de residentes em Maceió, restando 401 óbitos, reduzindo o número informado pelo SIM em 11,1\%. As perdas do estudo, DO extraviadas na Secretaria Municipal de Saúde de Maceió, prontuários não localizados ou incompletos, num total de $14,7 \%$ (59/401), tiveram suas características comparadas aos óbitos estudados, sem apresentar diferenças estatisticamente significantes.

A Secretaria Municipal de Saúde de Maceió disponibilizou cópias das DO preenchidas pelos médicos e acesso aos registros do SIM digitados por técnicos, após codificação e aplicação do programa seletor de causa básica à DO. As causas básicas informadas na DO e no SIM foram confrontadas com a causa básica obtida após análise dos prontuários mãe-neonato, acessados nos arquivos dos hospitais onde ocorreram os eventos.

A análise dos prontuários obedeceu ao critério para investigação de causa básica, utilizado pelo Núcleo de Epidemiologia, Instituto Materno-Infantil Professor Fernando Figueira (NEPI/IMIP), protocolojáutilizadoporCoutinho 17 no Recife, Estado de Pernambuco, e Vanderlei et al. 18. Foram resgatadas informações relativas à gestação e assistência ao parto, evolução clínica do neonato no hospital, incluindo consulta aos livros de registros, fichas de transferência e as informações na unidade de destino. Os exames complementares, dados de necropsia e histopa- 
tológico da placenta, quando realizados, subsidiaram o estudo.

O preenchimento do formulário da pesquisa, que resultou em novas condições e causas da morte, foi realizado separadamente por duas neonatologistas, após treinamento prévio, de acordo com as rotinas estabelecidas, padronizando a técnica de investigação de causa básica, variável do estudo. A análise dos prontuários foi realizada sem conhecimento da causa da DO ou da análise realizada por cada observadora, permitindo avaliar a concordância interobservadoras.

Ao final, os diagnósticos discordantes entre as observadoras foram revisados por ambas. Duas codificadoras com vínculo à Secretaria Estadual de Saúde de Pernambuco, e treinadas pelo Centro Brasileiro de Classificação de Doenças (CBCD) codificaram então a causa básica dos prontuários e recodificaram as DO seguindo as regras do Programa Seletor de Causas Básicas, utilizado pelo SIM. Obteve-se uma única causa básica por óbito (CB-Prontuário) considerada o "padrão-referência" na pesquisa, a ser comparada com o SIM (CB-SIM). A codificação da causa básica seguiu a CID-10 9, capítulos XVI e XVII, em três e quatro dígitos (totalizando 23 grupos), posteriormente reagrupados em sete grupos: grupo 1 - fatores materno-placentários e ligados ao parto, onde foram incluídas as doenças codificadas no CID-10 (P00-P04 e os traumas de parto, códigos P10-P15); grupo 2 - prematuridade, código P07; grupo 3 - hipóxia, agrupando afecções codificadas (P20-P21), sendo incluída a síndrome de aspiração meconial, código P24; grupo 4- desconforto respiratório do recém-nascido (código P22); grupo 5 - infecções perinatais, incluídas doenças codificadas (P36-P39), acrescidas da pneumonia neonatal (P23) e sífilis congênita (A50); grupo 6 - malformações congênitas, incluídas malformações letais codificadas entre Q00-Q99; grupo 7 - outras causas, outros códigos de afecções perinatais.

Definida a causa básica, procedeu-se à análise de redutibilidade. A classificação da Fundação SEADE modificada ${ }^{6}$ agrupa por causas todas as doenças constantes na CID-10 em três categorias: os óbitos redutíveis (por imunoprevenção; por adequado controle da gravidez; por adequada atenção ao parto; por diagnóstico e tratamento precoces, e por parcerias com outros setores), os óbitos não redutíveis e as causas mal definidas. Para aprimorar a análise dos eventos por esta classificação, os óbitos foram estratificados por peso ao nascer em duas categorias: $<2.500 \mathrm{~g}$ $\mathrm{e} \geq 2.500 \mathrm{~g}$

$\mathrm{Na}$ análise do óbito neonatal pela classificação de Wigglesworth 7 modificada são preconizados cinco agrupamentos de causas: grupo
1 - óbitos antes do trabalho de parto; grupo 2 - malformações congênitas em neomortos e natimortos; grupo 3 - condições associadas à prematuridade ou imaturidade; grupo 4 - óbitos por trauma ou asfixia; grupo 5 - condições específicas e outras. Há necessidade de detalhamento de informações como idade em horas no óbito, peso, idade gestacional e evidência de asfixia, devendo esta classificação ser usada para estudo de prontuários.

A tabulação, a validação por dupla entrada de dados e a análise estatística foram realizadas no programa Epi Info 6.04 (Centers for Disease Control and Prevention, Atlanta, Estados Unidos). A confiabilidade ou reprodutibilidade da causa básica no SIM foi aferida pela concordância simples e ajustada pelo indicador de kappa (< 0,00 ruim; 0,00-0,20 fraca; 0,21-0,40 sofrível; 0,41-0,60 regular; 0,61-0,80 boa; 0,81-0,90 ótima; 1,0 perfeita) 19 e seus intervalos de confiança, com significância estatística de $95 \%$. A análise da validade ou acurácia do SIM, grau em que este sistema apresenta a verdadeira causa básica ou o quanto se afasta desta, foi obtida comparandoo com a causa básica resultante da análise dos prontuários (padrão-referência) e aferida através da sensibilidade ou taxa de detecção e seus intervalos de confiança (IC95\%). Diferenças relativas foram utilizadas para mensurar e comparar a magnitude de diferenças encontradas entre o padrão-referência, DO e SIM.

A pesquisa foi autorizada pelas instituições e aprovada pelo Comitê de Ética em Pesquisa da Universidade Federal de Alagoas em 25 de novembro de 2003 (processo no ${ }^{\circ}$ 009571/2003-10).

\section{Resultados}

As características da população da pesquisa foram definidas a partir dos 337 prontuários estudados, dos quais 22,7\% (76/337) tinham peso ao nascer 2.500g; 55,4\% (187/337) sexo masculino; $53,6 \%(181 / 337)$ faleceram nas primeiras 24 horas de vida; $65 \%$ (219/337) nasceram de parto não operatório; $18,6 \%(63 / 337)$ eram a termo, com idade gestacional 37 semanas. As mães tinham idade $\leq 19$ anos em 31,5\% (106/337) casos. A concordância entre as observadoras na análise de prontuários foi $91,5 \%$ (308/337) para quatro dígitos.

As principais causas básicas identificadas no estudo (Tabela 1) foram os fatores maternos e ligados ao parto (códigos P00-P04), em 46,6\% (157/337) dos prontuários, e 7,1\% (24/337) no SIM; os transtornos respiratórios e cardiovasculares específicos (códigos P20-P29) identificados em 22\% (75/337) prontuários, e 59,6\% (201/337) 
Distribuição das causas básicas de óbitos neonatais por grupos de doenças, após análise de prontuários e Sistema de Informações sobre Mortalidade (SIM). Maceió, Alagoas, Brasil, 2001-2002.

\begin{tabular}{|c|c|c|c|c|c|}
\hline \multirow[t]{3}{*}{ Agrupamentos por capítulos CID-10 - causas básicas } & \multicolumn{4}{|c|}{ Causa básica } & \multirow{3}{*}{$\begin{array}{c}\text { Diferença } \\
\text { relativa } \\
\text { (prontuários } \\
\text { /SIM) } \\
\%\end{array}$} \\
\hline & \multicolumn{2}{|c|}{ Prontuário (CB-AP) } & \multicolumn{2}{|c|}{ SIM (CB-SIM) } & \\
\hline & $\mathbf{n}$ & $\%$ & $\mathbf{n}$ & $\%$ & \\
\hline Fatores maternos e complicações da gravidez; trabalho de parto, parto (P00-P04) & 157 & 46,6 & 24 & 7,1 & $-84,7$ \\
\hline Complicações ligadas à duração da gestação e crescimento fetal (P05-P08) & 11 & 3,3 & 16 & 4,7 & $+45,4$ \\
\hline Traumatismo no nascimento (P10-P15) & 2 & 0,6 & 2 & 0,6 & - \\
\hline Transtornos respiratórios e cardiovasculares específicos perinatais (P20-P29) & 75 & 22,2 & 201 & 59,6 & $+168,0$ \\
\hline Infecções específicas do período perinatal e sífilis (P35-P39 e A50) & 37 & 11,0 & 47 & 13,9 & $+27,0$ \\
\hline Transtornos hemorrágicos e hematológicos do recém-nascido (P50-P61) & 5 & 1,5 & 5 & 1,5 & - \\
\hline Transtornos endócrinos e metabólicos do recém-nascido (P70-P74) & - & - & 1 & 0,3 & $+100,0$ \\
\hline Transtornos do aparelho digestivo (P75-P78) & - & - & 1 & 0,3 & $+100,0$ \\
\hline Comprometimento do tegumento e regulação térmica (P80-P83) & - & - & 2 & 0,6 & $+100,0$ \\
\hline Outros transtornos (P90-P96) & 2 & 0,6 & - & - & $-100,0$ \\
\hline Malformações congênitas (Q00-Q99) & 48 & 14,2 & 35 & 10,3 & $-27,0$ \\
\hline Causas mal definidas (R00-R99) & - & - & 1 & 0,3 & $+100,0$ \\
\hline Outras CID & - & - & 2 & 0,6 & $+100,0$ \\
\hline Total & 337 & 100,0 & 337 & 100,0 & - \\
\hline
\end{tabular}

no SIM. Houve um aumento de diagnóstico de $554,2 \%$ dos óbitos por fatores maternos e complicações da gravidez e parto na análise de prontuários, não notificados corretamente ao SIM.

A concordância entre prontuários e o SIM (Tabela 2), em causas maternas e do parto, foi de $53,1 \%$, com sensibilidade de $8,8 \%$, sendo maior em prematuridade $(95,2 \%)$, com sensibilidade de $44,4 \%$. Na análise ajustada observou-se uma concordância fraca em causas maternas, assim como concordância fraca até sofrível para infecções, prematuridade, desconforto respiratório, outras causas de óbito e hipóxia.

Após codificação da causa básica em quatro dígitos, a gravidez múltipla e ruptura prematura de membranas corresponderam a 6,1\% (21/337) óbitos, e doença hipertensiva específica da gestação a 5\% (17/337) dos óbitos nos prontuários. Causas intermediárias ou terminais foram elencadas como causas básicas, destacando-se óbitos por asfixia grave em 7,3\% (25/337); infecções inespecíficas $6,4 \%(22 / 337)$, e doença de membrana hialina 8,7\% (30/337). Foi detectado elevado percentual de sífilis congênita nos prontuários, 4,1\% (14/337), dos quais os médicos só relataram na DO 2,3\% (9/337) de ocorrências, diferença relativa de $-35,7 \%$, quando o SIM registrou $0,7 \%$ (3/337) de óbitos, com diferença relativa de $-78,6 \%$.
Na Tabela 3, os acréscimos relativos ao grupo 2 (prematuridade) no SIM, corresponderam, quando analisados até o quarto dígito, ao grupo "outros prematuros". A insuficiência respiratória, que representou $10,4 \%$ dos casos (35/337), foi codificada pelo SIM, principalmente quando os médicos haviam definido prematuridade como causa básica. Dentre as falhas encontradas no SIM predominaram códigos inapropriados (códigos D69.6, J96.0, K42.9 e R99), uso incorreto de códigos como prematuridade, sem a observância da precedência do peso à idade gestacional, justificada em alguns casos pela omissão do peso do neonato na DO, uso abusivo de codificações inespecíficas e seleção incorreta de causa básica, como hipospádia (código Q54.9), agravando a inadequação deste sistema.

A análise de redutibilidade pela classificação da Fundação SEADE modificada (Tabela 4) evidenciou elevado percentual de causas redutíveis, principalmente quando da análise e definição adequada da causa básica aplicada aos prontuários, correspondendo a 76,1\% dos óbitos (256/337). Analisando sob a ótica da causa básica encontrada nas DO e no SIM, o percentual de causas redutíveis foi bem menor, chegando a 59,8\% (201/337) no SIM e 58,8\% (198/337) nas DO.

Nos prontuários, o maior número de causas redutíveis ocorreu por inadequado controle da 
Concordância entre agrupamentos de causas básicas entre prontuários e Sistema de Informações sobre Mortalidade (SIM), em óbitos neonatais precoces. Maceió, Alagoas, Brasil.

\begin{tabular}{lccc}
\hline Causas básicas & $\begin{array}{c}\text { Concordância } \\
\text { simples (\%) }\end{array}$ & $\begin{array}{c}\text { Sensibilidade } \\
\text { (IC95\%) }\end{array}$ & $\begin{array}{c}\text { Kappa * } \\
\text { (IC95\%) }\end{array}$ \\
\hline Grupo 1: causas maternas & 53,1 & $8,8(5,0-14,5)$ & $0,02(-0,04-0,08)$ \\
Grupo 2: prematuridade & 95,2 & $44,4(15,3-77,3)$ & $0,31(0,21-0,41)$ \\
Grupo 3: hipóxia & 75,6 & $46,5(31,5-62,2)$ & $0,19(0,09-0,29)$ \\
Grupo 4: desconforto respiratório & 67,7 & $60,0(49,7-76,8)$ & $0,14(0,06-0,22)$ \\
Grupo 5: infecções & 81,0 & $39,5(24,5-56,6)$ & $0,22(0,11-0,32)$ \\
Grupo 6: malformações congênitas & 92,5 & $60,4(45,3-73,9)$ & $0,65(0,55-0,75)$ \\
Grupo 7: outras causas & 92,8 & $22,2(3,9-59,8)$ & $0,11(0,01-0,21)$ \\
\hline
\end{tabular}

Nota: concordância geral - prontuário versus SIM = 31,4; kappa = 0,17 (IC95\%: 0,13-0,21).

Grupo 1: P00-P04 e P10-P15; grupo 2: P07; grupo 3: P20-P21 e P24; grupo 4: P22; grupo 5: P23, P36-P39 e A50; grupo 6:

Q00-Q99; grupo 7: outros códigos dos capítulos XVI e XVII da CID-10 9.

* Indicador kappa (<0,00 ruim; 0,00-0,20 fraca; 0,21-0,40 sofrível; 0,41-0,60 regular; 0,61-0,80 boa; 0,81-0,90 ótima;

1,0 perfeita) 19

gravidez, 40,2\% (135/337). No SIM, foram mais freqüentes óbitos redutíveis por diagnóstico e tratamento precoces, 37,9\% (128/337). Óbitos não redutíveis ocorreram em 39,9\% (134/337) no SIM, proporcionalmente superiores aos prontuários, 23,3\% (79/337). A análise dos prontuários e DO não detectou óbitos por causas redutíveis por parcerias com outros setores: causas infecciosas, neoplasias, AIDS e outras doenças virais, deficiências nutricionais, algumas anomalias congênitas e causas externas, ocorrendo um caso no SIM por erro de codificação. A concordância simples quanto à classificação da Fundação SEADE modificada entre prontuários e DO foi $41 \%$ (sofrível) e entre prontuários e SIM foi de 33\% (fraca). A concordância entre DO e o SIM foi $83 \%$ (sofrível).

A utilização da classificação da Fundação SEADE modificada nos óbitos distribuídos por peso, com ponto de corte em $2.500 \mathrm{~g}$ (Tabela 4), permitiu a detecção de elevado percentual $69,2 \%$ (54/336) de óbitos redutíveis em neonatos de peso $\ddagger 2500$ g, evidenciando a ocorrência de óbitos por inadequação de assistência numa faixa ponderal completamente viável, considerados eventos-sentinela da assistência perinatal no município. Destes, 24,3\% (19/78) seriam redutíveis por adequada atenção ao parto.

Na análise pela classificação de Wigglesworth modificada (Tabela 5), verificaram-se óbitos por malformações (14\%) passíveis de intervenção e rastreamento precoces; imaturidade (47\%) e fatores correlatos redutíveis por pré-natal e assistência neonatal adequados; óbitos por asfixia
(25,6\%) redutíveis e influenciados pela atenção adequada ao parto e outras causas específicas neonatais $(13,4 \%)$.

\section{Discussão}

Os óbitos neonatais ocorreram em percentual importante nas primeiras 24 horas de vida, em pré-termos, o que está de acordo com resultados de outras pesquisas realizadas em capitais brasileiras 11,12,20. Esse achado reflete o perfil observado para esses óbitos neonatais precoces desde a primeira metade da década de 1980. Com o desenvolvimento de tecnologias capazes de aumentar a sobrevida (UTI neonatal) para esses recém-nascidos, e com o aumento da cobertura de pré-natal, apesar de ainda inadequado em qualidade, há maior sobrevida nos primeiros sete dias de vida de crianças graves. Além disso, há sobrevivência de alguns recém-nascidos que anteriormente seriam óbitos fetais, com outras medidas de assistência ao parto, apesar de ainda insuficientes para uma redução importante do quadro geral da mortalidade neonatal 20 .

A causa básica de óbito é uma das mais relevantes informações disponíveis na DO. A sua validação, análise de sua concordância pela comparação com um padrão-ouro, ou padrão de referência permite avaliar a fidedignidade deste instrumento (DO) e coerência dos indicadores gerados com base neste banco de dados nacional. As causas maternas, na maioria evitáveis, são as mais freqüentes causas básicas e os 
Freqüência das principais causas básicas de óbitos neonatais precoces e diferenças relativas entre prontuários, e Sistema de Informações sobre Mortalidade (SIM). Maceió, Alagoas, Brasil, 2001-2002.

\begin{tabular}{|c|c|c|c|}
\hline \multirow[t]{3}{*}{ Causa básica - CID-10 (4 dígitos) } & \multicolumn{2}{|c|}{ Causa básica } & \multirow{3}{*}{$\begin{array}{c}\text { Diferença relativa } \\
\text { (prontuário/SIM) } \\
\%\end{array}$} \\
\hline & Prontuários & SIM & \\
\hline & $N=337$ & $N=337$ & \\
\hline \multicolumn{4}{|l|}{ Grupo 1: fatores materno-placentários e ligados ao parto } \\
\hline Doença hipertensiva específica da gestação (P00.0) & 17 & 2 & $-88,2$ \\
\hline Infecção do trato urinário (P00.8) & 16 & 2 & $-87,5$ \\
\hline Incompetência istmo-cervical (P01.0) & 11 & - & $-100,0$ \\
\hline Gemelaridade (P01. 5) & 19 & - & $-100,0$ \\
\hline Placenta prévia (P02.0) & 6 & 1 & $-83,3$ \\
\hline Descolamento prematuro placenta / hemorragia (P02. 1) & 12 & 5 & $-58,3$ \\
\hline Corioamnionite (P02. 7) & 15 & 1 & $-93,3$ \\
\hline Uso de drogas abortivas (P04. 1) & 6 & - & $-100,0$ \\
\hline \multicolumn{4}{|l|}{ Grupo 2: prematuridade } \\
\hline Recém-nascido muito baixo peso < 999g (P07.0) & 9 & - & - \\
\hline Outros prematuros (P07.1) & 1 & 15 & $+1400,0$ \\
\hline \multicolumn{4}{|l|}{ Grupo 3: hipóxia, síndrome de aspiração meconial } \\
\hline Hipóxia intra-uterina (P20.0) & 1 & 3 & $+200,0$ \\
\hline Hipóxia intra-útero não especificada (P20.9) & 2 & 1 & $-50,0$ \\
\hline Asfixia grave ao nascer (P21.0) & 24 & 10 & $-58,3$ \\
\hline Asfixia ao nascer, não especificada (P21.9) & 3 & 10 & $+233,3$ \\
\hline Síndrome de aspiração meconial (P24.0) & 5 & 7 & $+40,0$ \\
\hline \multicolumn{4}{|l|}{ Grupo 4: desconforto respiratório do recém-nascido } \\
\hline Doença da membrana hialina (P22.0) & 30 & 108 & $+260,0$ \\
\hline Insuficiência respiratória do recém-nascido (P28.5) & 1 & 35 & $+3400,0$ \\
\hline Outros distúrbios respiratórios (P28.9) & 2 & 1 & $-100,0$ \\
\hline \multicolumn{4}{|c|}{ Grupo 5: infecções perinatais, pneumonia neonatal, sífilis congênita } \\
\hline Pneumonia congênita não especificada (P23.9) & 1 & 9 & $+800,0$ \\
\hline Septicemia (P36. 9) & 1 & 28 & $+1300,0$ \\
\hline Sífilis (A 50.0) & 14 & 3 & $-78,6$ \\
\hline \multicolumn{4}{|l|}{ Grupo 6: malformações congênitas, malformações letais } \\
\hline Outras malformações e alterações esqueléticas (Q87.5) & 1 & 8 & $+700,0$ \\
\hline \multicolumn{4}{|l|}{ Grupo 7: outras causas, outros códigos perinatais } \\
\hline Outras causas (outras CID) & 120 & 73 & - \\
\hline
\end{tabular}

principais desencadeantes de óbitos neonatais, nos países em desenvolvimento 21,22 . Nos países desenvolvidos, as malformações congênitas estão em primeiro lugar, configurando um mínimo irredutível 1.

O preenchimento das condições e causas da morte neonatais tem peculiaridades, requerendo atenção do médico, principalmente nos eventos desencadeantes do nascimento e na evolução do neonato para o óbito, em sua maioria gerada na gestação, no trabalho de parto ou ainda, na assistência recebida pelo binômio mãe-bebe.

A prematuridade, a asfixia e a insuficiência respiratória são diagnósticos impróprios à causa básica, porque sua ocorrência pressupõe a existência de uma causa precedente, sendo esta a causa básica 3,17 . A prematuridade, para ser considerada causa básica deve ser a única afecção diagnosticada, para a qual nenhuma outra causa foi relatada 17. Confundir causa terminal com causa básica é também um erro freqüentemente cometido pelos médicos ${ }^{3}$ no preenchimento das DO e muito encontrado neste estudo.

Eventos como doença hipertensiva específica da gestação, placenta prévia, descolamento prematuro de placenta, hemorragia, corioamnionite e até uso voluntário de drogas abortivas pelas mães, consideradas causas maternas, foram de- 
Distribuição dos óbitos neonatais precoces, de acordo com a classificação da Fundação Sistema Estadual de Dados (Fundação SEADE) modificada 6 comparando as causas básicas em prontuários, e Sistema de Informações sobre Mortalidade (SIM), com e sem estratificação de peso ao nascer. Maceió, Alagoas, Brasil, 2001-2002.

\begin{tabular}{|c|c|c|c|c|c|c|c|c|c|c|}
\hline \multirow{5}{*}{$\begin{array}{l}\text { Classificação da Fundação } \\
\text { SEADE modificada }\end{array}$} & \multicolumn{2}{|c|}{ DO } & \multicolumn{2}{|c|}{ SIM } & \multicolumn{6}{|c|}{ Prontuário $(\mathrm{N}=336)$ * } \\
\hline & \multirow[b]{4}{*}{$\mathbf{n}$} & \multirow[b]{4}{*}{$\%$} & \multirow[b]{4}{*}{$\mathbf{n}$} & \multirow[b]{4}{*}{$\%$} & \multirow{2}{*}{\multicolumn{2}{|c|}{$\begin{array}{c}\text { Sem estratificação } \\
\text { de peso }\end{array}$}} & \multirow{2}{*}{\multicolumn{4}{|c|}{$\begin{array}{c}\text { Com estratificação } \\
\text { de peso }\end{array}$}} \\
\hline & & & & & & & & & & \\
\hline & & & & & \multirow[b]{2}{*}{$\mathrm{n}$} & \multirow[b]{2}{*}{$\%$} & \multicolumn{2}{|c|}{$<2.500 \mathrm{~g}$} & \multicolumn{2}{|c|}{$\geq 2.500 \mathrm{~g}$} \\
\hline & & & & & & & $\mathrm{n}$ & $\%$ & $\mathrm{n}$ & $\%$ \\
\hline 1. Óbitos redutíveis por & 233 & 58,8 & 240 & 59,8 & 261 & 76,1 & 202 & 78,3 & 54 & 69,2 \\
\hline 1A. Imunoprevenção & - & - & - & - & - & - & - & - & - & - \\
\hline \multicolumn{11}{|l|}{ 1B. Adequado controle } \\
\hline da gravidez & 52 & 13,1 & 36 & 9,0 & 138 & 40,2 & 120 & 46,5 & 13 & 16,7 \\
\hline \multicolumn{11}{|l|}{ 1C. Adequada atenção } \\
\hline ao parto & 38 & 9,6 & 52 & 13,0 & 83 & 24,2 & 64 & 24,8 & 19 & 24,3 \\
\hline \multicolumn{11}{|l|}{ 1D. Diagnóstico e } \\
\hline tratamento precoces & 143 & 36,1 & 151 & 37,7 & 40 & 11,7 & 18 & 7,0 & 22 & 28,2 \\
\hline 1E. Parcerias com outros setores & - & - & 1 & 0,2 & - & - & - & - & - & - \\
\hline 2. Não redutíveis & 160 & 40,4 & 160 & 39,9 & 80 & 23,2 & 55 & 21,3 & 23 & 29,5 \\
\hline 3. Mal definidas & 3 & 0,8 & 1 & 0,2 & 2 & 0,6 & 1 & 0,4 & 1 & 1,3 \\
\hline Total & 396 & 100,0 & 401 & 100,0 & 343 & 100 & 258 & 100,0 & 78 & 100,0 \\
\hline
\end{tabular}

* Excluídos óbitos < 500g e peso não aferido.

DO: declaração de óbito.

tectados neste e em outros estudos 11,23, como determinantes do nascimento de prematuros, estando relatadas nos prontuários, mas não sendo sequer citados pelos médicos, ao redigirem as DO. Estes neonatos prematuros, evoluindo com desconforto respiratório, possivelmente doença da membrana hialina, e vindo a falecer, tinham como causas de óbito assinaladas pelos médicos prematuridade e doença de membrana hialina, levando ao sobre-diagnóstico destas condições nas DO e SIM, omitindo a causa básica.

A avaliação de concordância da causa básica pode ser feita utilizando-se a codificação com três dígitos, englobando várias causas de óbito de mesma origem, a exemplo do código P07, onde estão diferentes estágios e códigos de prematuridade e baixo peso. A análise da causa codificada com quatro dígitos geralmente propicia uma codificação mais precisa do evento, apesar de estarem incluídos em todos os grupos de doenças perinatais, códigos utilizados quando a causa não pode ser mais bem definida, a exemplo de P07.3 - outros recém-nascidos pré-termo.

A baixa concordância entre os prontuários e o SIM reflete a inadequação do preenchimento da DO, também relatada por Borrell et al. 24, em Barcelona, que encontraram concordância de $40,1 \%$ em três dígitos, após revisão de prontuários. Por outro lado, apesar de ter sido considerado padrão de referência, e ser utilizado como fonte de informação para revisão das DO e conseqüente revisão do SIM, o prontuário pode apresentar em diferentes serviços padrões diversos de qualidade. Em relação ao estudo, poucos prontuários apresentaram inadequação de preenchimento, contribuindo para que causas intermediárias ou terminais fossem elencadas como causa básica, destacando-se óbitos por asfixia grave, infecções inespecíficas. Talvez esse fato tenha sido verificado por tratar-se de óbitos ocorridos em hospitais terciários, com algum padrão estabelecido para preenchimento e possibilidade de resgate das informações em livros de registros de sala de parto, e outros registros hospitalares.

A aferição de sensibilidade ou taxa de detecção também é uma forma de verificar a capacidade da DO e SIM em discriminar corretamente a causa básica, ao compará-las a um padrão de referência, a exemplo do formulário preenchido a partir dos prontuários, com critérios dos CBCD. A maior sensibilidade do SIM comparado aos prontuários esteve nas malformações congênitas. É possível que esse resultado expresse um viés local do método de codificação: maior facilidade de classificação para esse grupo de doenças. Enquanto a diferença de causas encontradas 
Distribuição dos óbitos neonatais precoces de acordo com a classificação de Wigglesworth modificada por Keeling et al. 8 e com o peso ao nascer, assistência em UTI, idade gestacional e idade no óbito. Maceió, Alagoas, Brasil, 2001-2002.

\begin{tabular}{|c|c|c|c|c|c|c|c|c|c|c|}
\hline \multirow[t]{3}{*}{ Óbitos neonatais } & \multicolumn{8}{|c|}{ Classificação de Wigglesworth modificada por Keeling et al. 8} & \multicolumn{2}{|c|}{ Total } \\
\hline & \multicolumn{2}{|c|}{ Malformações } & \multicolumn{2}{|c|}{ Imaturidade } & \multicolumn{2}{|c|}{ Asfixia } & \multicolumn{2}{|c|}{ Outras específicas } & \multirow[b]{2}{*}{$\mathbf{n}$} & \multirow[b]{2}{*}{$\%$} \\
\hline & $\mathrm{n}$ & $\%$ & $\mathrm{n}$ & $\%$ & $\mathrm{n}$ & $\%$ & $\mathrm{n}$ & $\%$ & & \\
\hline \multicolumn{11}{|l|}{ Peso ao nascer } \\
\hline$<2.500 \mathrm{~g}$ & 25 & 53,2 & 154 & 97,4 & 59 & 68,6 & 20 & 44,4 & 258 & 76,8 \\
\hline$\geq 2.500 \mathrm{~g}$ & 22 & 46,8 & 4 & 2,6 & 27 & 31,4 & 25 & 55,6 & 78 & 23,2 \\
\hline Total & 47 & 100,0 & 158 & 100,0 & 86 & 100,0 & 45 & 100,0 & 336 * & 100,0 \\
\hline \multicolumn{11}{|c|}{ Assistência hospitalar ao neonato } \\
\hline Com UTI & 30 & 63,8 & 126 & 79,7 & 51 & 59,3 & 25 & 55,6 & 232 & 69,0 \\
\hline Sem UTI & 17 & 36,2 & 32 & 20,3 & 35 & 40,7 & 20 & 44,5 & 104 & 31,0 \\
\hline Total & 47 & 100,0 & 158 & 100,0 & 86 & 100,0 & 45 & 100,0 & 336 * & 100,0 \\
\hline \multicolumn{11}{|l|}{ Idade gestacional } \\
\hline Pré-termo & 22 & 56,4 & 150 & 100,0 & 40 & 59,7 & 16 & 44,4 & 228 & 78,0 \\
\hline Termo & 17 & 43,6 & - & - & 27 & 40,3 & 20 & 55,6 & 64 & 21,9 \\
\hline Total & 39 & 100,0 & 150 & 100,0 & 67 & 100,0 & 36 & 100,0 & 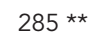 & 100,0 \\
\hline \multicolumn{11}{|c|}{ Idade no óbito (horas) } \\
\hline$\leq 24$ & 28 & 61,0 & 83 & 51,2 & 47 & 55,3 & 21 & 47,7 & 179 & 53,91 \\
\hline$>24$ & 18 & 39,0 & 74 & 48,8 & 38 & 44,7 & 23 & 52,3 & 153 & 46,1 \\
\hline Total & 46 & 100,0 & 162 & 100,0 & 85 & 100,0 & 44 & 100,0 & 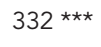 & 100,0 \\
\hline Total & 47 & 14,0 & 158 & 47,0 & 86 & 25,6 & 45 & 13,4 & 336 * & 100,0 \\
\hline
\end{tabular}

* Excluídos óbitos < 500g e peso não aferido;

** Excluídos óbitos com idade gestacional não informada;

*** Excluídos óbitos < 500g e não aferido e idade não informada.

entre DO e prontuários podem refletir o desconhecimento médico acerca do preenchimento da DO, as diferenças encontradas entre o SIM e DO são passíveis de refletir erros de codificação e/ou digitação no sistema, incluindo o uso de códigos inadequados ao período neonatal.

Estudos anteriores reforçam a importância das causas maternas e enfatizam o desconhecimento médico no correto preenchimento da DO, refletindo-se na codificação no SIM e o excesso de diagnósticos imprecisos, propiciando inconsistência estatística 9,17,25,26. O elevado percentual de causas maternas nos prontuários encontrados neste estudo é compatível com outras pesquisas realizadas no Brasil 11,12,16,21,27. Entretanto, estudo realizado no Rio de Janeiro 28 apresentou percentuais bem mais elevados de asfixia. Coutinho 17, em estudo prospectivo, obteve maior eficiência no rastreamento das causas maternas, registrando maior percentual $(52,3 \%)$ em estudos brasileiros.

Grande parte dos óbitos neonatais pode ser evitada por assistência adequada. Quantificá-los por critérios que analisem este potencial permi- te visualizar onde e como as ações falharam 11. Nessa ótica, foram utilizadas neste estudo, a exemplo de outros autores 12,17, as classificações da Fundação SEADE e de Wigglesworth modificadas, evidenciando elevado percentual de óbitos redutíveis. Não foi detectado nenhum caso redutível por imunoprevenção, possivelmente por subdiagnóstico, principalmente em hepatite $B$ e rubéola congênita, cujos óbitos podem ser mais tardios.

O percentual de óbitos redutíveis foi maior nos prontuários que no SIM, devido à correta definição da causa básica propiciada pela análise dos prontuários, evidenciando a importância do preenchimento adequado das condições e causas da morte na aplicação de classificações que visem avaliar redutibilidade, a partir da causa básica. Houve diferenças substanciais no foco de atenção para redutibilidade: nos prontuários predominaram causas que indicavam necessidade de adequado controle da gravidez, enfocando possíveis falhas na qualidade do pré-natal e refletindo o descontrole de doenças inadmissíveis no século XXI, como a sífilis congênita e 
doenças maternas ou complicações como doença hipertensiva específica da gestação, diabetes e infecções.

A análise do SIM na mesma ótica remete ao diagnóstico e tratamento precoces das causas neonatais, indicando dificuldade de acesso da população aos serviços, incluindo os de maior complexidade, como nas UTI. É possível que importante percentual de crianças com indicação de cuidados intensivos não tenha sido assistida em hospital com UTI neonatal.

Alguns autores têm preferido o uso da classificação de Wigglesworth à classificação da Fundação SEADE, considerando sua praticidade na análise dos óbitos 11,12. No presente estudo não foram observadas, a priori, diferenças substanciais nos percentuais de óbitos redutíveis entre as duas classificações. No entanto, observou-se que as características da classificação de Wigglesworth ao restringir seu uso ao ambiente hospitalar, não permitem que sua análise alcance a totalidade de aspectos e processos que antecederam e resultaram na assistência ao neonato. Desse modo, ficam excluídas as causas maternas, como a doença hipertensiva específica da gestação, infecções, diabetes e outros processos desencadeantes, apenas subentendidas, classificando em um mesmo grupamento, neonatos que vivenciaram experiências bastante diversas no seu processo de assistência, situações que nem sempre podem ser consideradas pertencentes a um mesmo grupo. Nos casos onde haja a visualização de causas mais evidentes e terminais pela não discriminação de situações desencadeantes, Cartlidge et al. 29 asseveram a possibilidade de incorreção de diagnóstico, o que só pode ser detectado pela necrópsia, procedimento de difícil acesso na maioria das cidades brasileiras.

A classificação da Fundação SEADE modificada se mostrou mais adequada, pois permite avaliações nos serviços hospitalares bem como a utilização dos dados de mortalidade em um sistema como o SIM, e, em níveis mais amplos de decisão e planejamento em saúde, permite a determinação de grupos de risco e a focalização de ações preventivas, principalmente quando incorpora a análise por faixas ponderais. Todavia, o uso de classificações mais robustas e com maior propriedade requer que o SIM apresente informações de boa qualidade, se reportando à verdadeira causa básica e sendo submetido re- gularmente às criticas e análises de consistência necessárias e previstas pelo próprio sistema.

A distribuição dos óbitos por peso, na classificação da Fundação SEADE modificada evidenciou elevado percentual de causas redutíveis em neonatos pesando $\geq 2.500 \mathrm{~g}$, permitindo demonstrar um maior refinamento desta classificação para indicar população de risco, potencializando sua capacidade para detecção de eventos-sentinela. As classificações baseadas na redutibilidade dos óbitos neonatais devem considerar o peso do neonato, variável importante entre os determinantes do óbito neonatal e capaz de modificar resultados, quando não considerada.

Outro aspecto de relevância é o preenchimento adequado dos prontuários materno-infantis como indicador da qualidade do atendimento ao binômio mãe-filho, como sugerem Campos et al. ${ }^{30}$, ressaltando a importância da análise conjunta dos prontuários da mãe e neonato, para um melhor reconhecimento da causa básica dos óbitos neonatais.

A não validação de endereços, a aceitação de DO sem endereço firmado, a ausência de crítica de inconsistências em causa básica e idade podem ter contribuído para falhas de classificação de óbitos e má qualidade do SIM. Ressalta-se que investir na confiabilidade e validade dos dados do SIM de Maceió permitirá sua adequada utilização e a incorporação da análise dos óbitos sob a ótica de prevenção na rotina da Secretaria Municipal de Saúde de Maceió, o que depende de informações confiáveis no SIM.

$\mathrm{O}$ treinamento no preenchimento da DO e dos profissionais ligados ao fluxo destes documentos é importante para que os dados do SIM possam ser utilizados em sua plenitude, traçando um perfil real da mortalidade infantil de Maceió, onde investimentos dos gestores de saúde, principalmente na qualidade da atenção à saúde da gestante e no acompanhamento ao parto, certamente se refletirão em diminuição no coeficiente de mortalidade infantil do município e do Estado.

A necessidade de dados fidedignos parece inquestionável, já que as principais exigências epidemiológicas de um sistema de informação são exatidão e consistência, necessitando compromisso dos gestores e profissionais, dos médicos aos digitadores, para melhoria na qualidade do SIM. 


\section{Resumo}

A análise da qualidade da informação sobre causas de óbitos neonatais no Brasil é extremamente relevante, permitindo verificar a magnitude de óbitos passíveis de redução e subsidiar políticas adequadas à sua diminuição. Esse estudo objetivou avaliar a confiabilidade e validade do Sistema de Informação sobre Mortalidade (SIM) na discriminação da causa básica de óbitos neonatais e definir percentuais de causas redutiveis. Foram analisadas causas básicas dos óbitos neonatais precoces hospitalares de Maceió, Alagoas, comparando as causas dos prontuários ao SIM, aferindo confiabilidade e validade. Para análise de redutibilidade compararam-se as classificações da Fundação SEADE e de Wigglesworth modificadas. Predominaram causas maternas nos prontuários e de transtornos respiratórios nas declarações de óbito e SIM. O percentual de óbitos redutíveis pode ser bem superior ao detectado no SIM, devido às imprecisões no preenchimento das declarações de óbito. De acordo com o SIM, os maiores problemas residem no diagnóstico e tratamento precoce de causas neonatais. Todavia, os resultados evidenciaram que os problemas mais prementes relacionamse a falhas no pré-natal e descontrole de doenças.

Mortalidade Neonatal; Causa Básica de Morte; Atestados de Óbito; Registros de Mortalidade

\section{Referências}

1. Lansky S, França E, Leal MC. Mortalidade perinatal e evitabilidade: revisão da literatura. Rev Saúde Pública 2002; 36:759-72.

2. Aerts DRGC. Investigação dos óbitos perinatais e infantis: seu uso no planejamento de políticas públicas de saúde. J Pediatr (Rio de J) 1997; 73: 364-6.

3. Sarinho SW, Coutinho SB, Accioly T, Menezes TF Abreu MAP, Lacerda N, et al. Mortalidade neonatal em Recife, PE, em 1998: causas básicas e grau do conhecimento dos neonatologistas acerca do preenchimento das declarações de óbito. Pediatria (São Paulo) 2001; 23:279-84.

\section{Colaboradores}

L. D. C. O. Pedrosa coordenou e participou da coleta de dados, realizou pesquisa bibliográfica, análise dos dados e redigiu o artigo. M. R. Ordonha contribuiu na coleta e análise dos dados e na redação do artigo. S. W. Sarinho contribuiu na elaboração e organização da pesquisa, na análise dos dados, redação e na revisão crítica do artigo.

\section{Agradecimentos}

As Dras. Maria José Guimarães e Lygia Carmem Vanderlei pela revisão crítica e assessoria científica, ao Dr. Ricardo A. A. Ximenes pela orientação na análise estatística.
4. Laurenti R. A análise da mortalidade por causa básica e por causas múltiplas. Rev Saúde Pública 1974; 8:421-35.

5. Organização Mundial da Saúde. Classificação estatística internacional de doenças e problemas relacionados à saúde, 10a revisão. v. 2. São Paulo: Centro Colaborador da OMS para a Classificação de Doenças em Português; 1995.

6. Fundação Sistema Estadual de Dados. Mortalidade infantil e desenvolvimento. Conjuntura Demográfica 1991; 14/15:49-50.

7. Wigglesworth JS. Monitoring perinatal mortality: a pathophysiological approach. Lancet 1980; 2:684-6. 
8. Keeling JW, MacGillivray I, Golding J, Wigglesworth J. Classification of perinatal death. Arch Dis Child 1989; 64:1345-51.

9. Organização Mundial da Saúde. Classificação estatística internacional de doenças e problemas relacionados à saúde, 10a revisão. v. 1. São Paulo: Centro Colaborador da OMS para a Classificação de Doenças em Português; 1995.

10. Ortiz LP. Características da mortalidade neonatal no estado de São Paulo [Tese de Doutorado]. São Paulo: Faculdade de Saúde Pública, Universidade de São Paulo; 1999.

11. Lansky S, França E, Leal MC. Mortes perinatais evitáveis em Belo Horizonte, Minas Gerais, Brasil, 1999. Cad Saúde Pública 2002; 18:1389-400.

12. Leite AJM, Marcopito LF, Diniz RLP, Silva AVSE, Souza LCB, Borges JC, et al. Mortes perinatais no município de Fortaleza, Ceará: o quanto é possível evitar? J Pediatr (Rio de J) 1997; 73:388-94.

13. Departamento de Informática do SUS. Indicadores de mortalidade 2004. Número de óbitos infantis (menores de 1 ano) por 1.000 nascidos vivos, Brasil, 1997-2002. http://tabnet.datasus.gov.br/ cgi/idb2004/c01.htm (acessado em 13/Dez/2004).

14. Organização Pan-Americana da Saúde. Métodos de investigação epidemiológica em doenças transmissíveis. v. 1. Brasília: Organização Pan-Americana da Saúde; 1997.

15. Turrini RNT, Santo AH. Infecção hospitalar e causas múltiplas de morte. J Pediatr (Rio de J) 2002; 78:485-90.

16. Guimarães MJB, Marques NM, Melo Filho DA, Szwarcwald CL. Condição de vida e mortalidade infantil: diferenciais intra-urbanos no Recife, Pernambuco, Brasil. Cad Saúde Pública 2003; 19:1413-24.

17. Coutinho SB. Mortalidade neonatal em cinco maternidades da cidade do Recife, 1994. Recife: Fundo das Nações Unidas para a Infância; 1996.

18. Vanderlei LC, Arruda BKG, Frias PG, Arruda S. Avaliação da qualidade de preenchimento das declarações de óbito em unidade terciária de atenção à saúde materno-infantil. Inf Epidemiol SUS 2002; 11:7-14.

19. Pereira MG. Aferição dos eventos. In: Pereira MG, organizador. Epidemiologia: teoria e prática. Rio de Janeiro: Editora Guanabara Koogan; 2000. p. 358-76.
20. Miura E, Failace LH, Fiori H. Mortalidade perinatal e neonatal no Hospital de Clínicas de Porto Alegre. Rev Assoc Med Bras 1997; 43:35-9.

21. Coutinho SB, Lima MC, Lira PIC. Estudo das causas de morte neonatal segundo o critério de evitabilidade do óbito. Revista Pediátrica de Pernambuco 1999; 11:1-2.

22. Szwarcwald CL, Leal MC, Castilho EA, Andrade CLT. Mortalidade infantil no Brasil: Belíndia ou Bulgária? Cad Saúde Pública 1997; 13:503-16.

23. Léon-López R, Gallegos-Machado B, EstévezRodríguez E, Rodríguez-García S. Mortalidad infantil. Análisis de un decenio. Rev Cuba Med Gen Integr 1998; 14:606-10.

24. Borrell KX, Ricart M, Bellart J, Plasencia A. Validation of perinatal causes of death in death certificates. An Esp Pediatr 1997; 47:410-6.

25. Formigli VLA, Silva LMV, Cerdeira AJP, Pinto CMF, Oliveira RSA, Caldas AC, et al. Avaliação da atenção à saúde através da investigação de óbitos infantis. Cad Saúde Pública 1996; 12 Suppl 2:S33-41.

26. Nobre LC, Victora CG, Barros FC, Lombardi C. Evaluation of the quality of information on the basic cause of death of infants in Rio Grande do Sul (Brazil). Rev Saúde Pública 1989; 23:207-13.

27. Leal MC, Szwarcwald CL. Evolução da mortalidade neonatal no Estado do Rio de Janeiro, Brasil (19791993): análise por causa segundo grupo de idade e região de residência. Cad Saúde Publica 1996; 12:243-52.

28. Carvalho ML, Silver LD. Confiabilidade da declaração da causa básica de óbitos neonatais: implicações para o estudo da mortalidade prevenível. Rev Saúde Pública 1995; 29:342-8.

29. Cartlidge PHT, Dawson AT, Stewart JH, Vujanic GM. Value and quality of perinatal and infant postmortem examinations: cohort analysis of 400 consecutive deaths. BMJ 1995; 310:155-8.

30. Campos MR, Leal MC, Souza Jr. PR, Cunha CB. Consistência entre fontes de dados e confiabilidade interobservador do Estudo da Morbi-mortalidade e Atenção Peri e Neonatal no Município do Rio de Janeiro. Cad Saúde Pública 2004; 20 Suppl 1: S34-43.

Recebido em 21/Jun/2006

Versão final reapresentada em 17/Jan/2007

Aprovado em 20/Abr/2007 